\title{
Emfiermenta

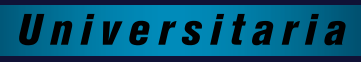

\section{Proceso de cuidado desde la óptica de familias mexicanas, urbanas y pobres}

\section{Healthcare process from the perspective of poor urban mexican families}

\section{Processo de cuidado desde a óptica de famílias mexicanas urbanas pobres}

\author{
R.M. Ostiguín-Meléndez ${ }^{a *}$, S. Bustamante-Edqén ${ }^{b}$ \\ a Escuela Nacional de Enfermería y Obstetricia, Universidad Nacional Autónoma de México, Ciudad de México, \\ México. \\ ${ }^{\mathrm{b}}$ Universidad de Trujillo, Perú
}

Recibido: 2 diciembre 2016

Aceptado: 10 enero 2018

\section{Resumen}

La familia ha sido vista como recurso, medio, contexto o elemento donde el cuidado de, entre y por sus integrantes adquiere sentido y poco se sabe sobre los mecanismos del cuidado familiar al interior, por lo que es importante desde marcos epistémicos específicos desvelar los elementos que participan; bajo esta perspectiva esta investigación es una contribución a la salud colectiva.

El objetivo fue explorar el cuidado familiar en población mexicana como proceso.

Metodología: El estudio fue cualitativo, descriptivo y exploratorio; se realizó en cinco familias mexicanas de la Ciudad de México que acuden a un dispensario médico. La información fue recabada con la guía de entrevista y notas de campo; el análisis fue temático.

Los resultados muestran que el cuidado familiar es un proceso autopoiético, en tanto que se establece como rasgo ontogenético, donde se aprende a cuidar en, para y por la familia transgeneracionalmente por medio de un acto de auto construcción que evita la importación indiscriminada de acciones de cuidado desde el exterior y se moviliza como bucle-recursivo, para asegurar la preservación de modelos familiares a través de un acto de adaptación que genera mecanismos de preservación del cuidado como rasgo que identifica al grupo, en donde sus valores determinan la acción cotidiana de cuidar en la familia.

Conclusiones: El cuidado familiar es un proceso autopoiético en bucle recursivo, en tanto que sus integrantes experimentan cambios constantes que preservan o no desde su propia ontogenia como familia que cuida.

*Autor para correspondencia, correo electrónico: omrm2011@gmail.com (R.M. Ostiguín-Meléndez) 
Palabras clave: Cuidado; apoyo familiar; enfermería; relaciones familiares; México

\section{Abstract}

The family has been seen as a resource, medium, context, and provider element, but little is known about its internal healthcare mechanisms. Because of this, it is important, from the specific epistemic frameworks, to unveil the elements involved in these processes. This study addresses the issue and contributes to the advancement of collective health.

The objective was to explore the Mexican family healthcare process.

Method: The study design was qualitative, descriptive, and exploratory. Five Mexico City families attending a medical facility were studied. Data were gathered through guided interviews and field notes. The analysis was thematic.

Results suggest that family healthcare is an ontogenetic-featured autopoietic process which is learned and transferred through generations as a cycle and in a self-constituting form in order to face and avoid external threats, thus preserving the family's unique functional model.

Conclusions: Considering the specific characteristics of healthcare within the poor urban Mexican families, one conclusion is that it is important to conduct further studies on the phenomenon in order to contribute to the improvement of health of these families.

Keywords: Care; family support; nursing; family relationships; Mexico.

\section{Resumo}

A família tem sido vista como recurso, médio, contexto ou elemento onde o cuidado de, entre e por seus integrantes adquire sentido e pouco se sabe sobre os mecanismos do cuidado familiar ao interior, pelo que é importante desde quadros epistémicos específicos desvelar os elementos que participam; sob esta perspectiva esta pesquisa é uma contribuição à saúde coletiva.

O objetivo foi explorar o cuidado familiar em população mexicana como processo.

Metodologia: O estudo foi qualitativo, descritivo e exploratório; realizou-se em cinco famílias mexicanas da Cidade do México que assistem a um dispensário médico. A informação foi coletada com a guia de entrevista e notas de campo; a análise foi temática. Os resultados mostram que o cuidado familiar é um processo autopoiético, enquanto se estabelece como traço ontogenético, onde se aprende a cuidar em, para e pela família transgeracionalmente, por médio de um ato de auto construção que evita a importação indiscriminada de ações de cuidado desde o exterior e mobiliza-se como espiral-recursivo, para assegurar a preservação de modelos familiares a través de um ato de adaptação que gera mecanismos de preservação do cuidado como traço que identifica ao grupo, onde seus valores determinam a ação cotidiana de cuidar na família. Conclusões: O cuidado familiar é um processo autopoiético em espiral- recursivo, enquanto seus integrantes experimentam câmbios constantes que preservam ou não desde sua própria ontogenia como família que cuida.

Palavras chave: Cuidado; apoio familiar; enfermagem; relações familiares; México.

\section{Introducción}

La familia mexicana contemporánea ha presentado modificaciones importantes en su estructura y dinámica en los últimos 30 años, desde el punto de vista sociodemográfico se ha producido gradualmente una disminución del tamaño promedio y ha transitado hacia la diversificación en la estructura familiar, lo que conlleva al cambio en los roles de sus integrantes, entre otras cosas. Destaca la creciente incorporación de la mujer al trabajo extra doméstico, el aumento de la participación cada día más activa de los hombres en el cuidado y un papel más activo de los hijos en las tareas al interior de la misma; aspectos que en mayor o menor grado determinan "una forma" de cuidar por parte de las familias ${ }^{1-5}$. 
En este marco el cuidado a la salud familiar se enfrenta en forma recurrente a procesos que le permitan cumplir con la responsabilidad de proveer condiciones de desarrollo para sus miembros; sin embargo, factores como la pobreza y la cronicidad determinan el qué y cómo se cuida la salud dentro y fuera del núcleo familiar.

Para el caso de México, donde, alrededor del 50 por ciento de la población se ubica en algún nivel de pobreza $^{2}$, es preciso tener presente que una parte importante de la población y por tanto de las familias, deben resolver sus necesidades con presupuestos altamente restringidos para la provisión de alimento, educación y servicios de salud, todos aspectos indispensables para el desarrollo y salud de una persona. Aunado a esta situación, está el hecho de que $68.4 \%$ de mexicanos carecen de acceso a servicios de salud ${ }^{2}$. En la Ciudad de México (CDMX) los dispensarios médicos generalmente cumplen con la provisión de atención a la salud en las zonas más pobres, tal es el caso de la delegación política Gustavo A. Madero, que ocupa el $2^{\circ}$ lugar en densidad de población en la CDMX, con una concentración de hogares familiares de tipo nuclear (7 de cada 10) ${ }^{3}$ y en donde se reporta que el 44\% de las familias carecen de algún tipo de servicio de salud, por lo que recurren a la atención en consultorios médicos de bajo costo o beneficencia ${ }^{4}$.

Por otro lado, la presencia cada vez más temprana de Enfermedades No Transmisibles como la diabetes y el cáncer ${ }^{5-7}$, conlleva frecuentemente a experimentar procesos de cronicidad saturados, de inversiones económicas y en especie (tiempo de cuidado) para el afrontamiento de la enfermedad, mismos que sin duda generan al interior de la familia procesos de cuidado singulares que desde su óptica tienen sentido.

Ante este escenario el cuidado familiar es un elemento de alto valor para las ciencias de la salud como la enfermería, en tanto que desde ahí las personas aprenden, desarrollan, promueven, modifican o crean formas de garantizar la salud de sus miembros. De este modo los valores y las creencias de los mexicanos respecto a sus familias continúan siendo altamente positivos, lo suficiente para que en el marco de la pobreza aún sea el punto primario de provisión de cuidados para el desarrollo de las personas ${ }^{8,9}$.

La familia "clásica" de hombre, mujer e hijos ha evolucionado lo suficiente como para hablar hoy de un grupo de individuos familiares o no, de géneros diferentes o iguales, con edades diversas y pertenecientes a varias generaciones con una corresponsabilidad en la provisión de uno o varios satisfactores de vida (alimentación, vestido, educación, etc.) y cuidado, mismo que se distribuye y desarrolla a partir del marco cultural donde existen y desde el cual sus prácticas tienen pertinencia; por lo que explorar y comprender la forma en que diversos elementos se articulan en el proceso de cuidado familiar desde la óptica de los protagonistas es altamente significativo para los profesionales de la enfermería, que de forma permanente se vinculan con ellos para mantener la salud individual o colectiva.

Al respecto del cuidado familiar, se reconoce que la aproximación se ha realizado desde múltiples teorías como la estructural funcional ${ }^{10,11}$, que la sitúa como una organización dada por sus miembros; la teoría general de sistemas, donde es visualizada como un sistema constituido por tres subsistemas: el micro, meso y macro sistema ${ }^{12}$ o la teoría de los sistemas familiares de Bowen, que la aborda como una unidad emocio$\mathrm{nal}^{13}$, por mencionar algunas. Sin embargo, los cuerpos teóricos arrojan atributos sustantivos en torno a la familia tales como su carácter central en procesos colectivos de los individuos, la coexistencia de personas vinculadas de diversas maneras: directa (consanguíneos), indirecta (amigos) o por una relación civil; la cohesión en términos de interacción entre sus integrantes y las relaciones afectivas, enfatiza su función principal, el bienestar de los individuos que la constituyen en donde la salud y su cuidado tienen sentido.

Los reportes sobre la familia y la cronicidad exponen al cuidado familiar en varios sentidos: el primero, en donde el individuo es visualizado como figura o sujeto de cuidado en el contexto del propio grupo familiar ${ }^{14}$, y el segundo, donde se enfoca al individuo-familia como unidad de cuidado ${ }^{15}$.

Desde hace más de una década se ha reconocido que el cuidado familiar es una asignatura pendiente desde la política pública en países latinoamericanos ${ }^{16}$; que la vivencia de cuidar no es homogénea en tanto que cada unidad básica tiene sus propios procesos ${ }^{17}$; que es necesario pensar en incluir a la familia en la prestación de cuidados, con respeto a sus propios procesos ${ }^{18}$ y que la experiencia de cuidar en la familia es un aspecto más de la vida y que forma parte de ella ${ }^{19}$; no obstante, poco se sabe sobre los mecanismos que 
promueven el cuidado familiar como proceso, que permiten perpetuar prácticas de cuidado, así como en su caso adaptarlas a los nuevos y constantes requerimientos de salud del grupo.

Algunos estudios más recientes enfocados en la capacidad autogestiva de la familia para atender la cronicidad $^{20,21}$, destacan que el conocimiento de la familia y sus dinámicas o procesos son sustantivas para cualquier intervención. Así, el estudio del cuidado familiar es sin duda un tema relevante y actual que debe ocupar la atención de enfermería.

A partir de este escenario es posible preguntarse cómo es el proceso de cuidado familiar en la población mexicana, por lo tanto, se pretende que los resultados abonen al campo de conocimiento de la familia y el cuidado en su interior, esto como referente para los profesionales de la enfermería contemporánea.

\section{Métodos}

La metodología en que la investigación se enmarcó fue cualitativa, en tanto que permitió recuperar desde la subjetividad de los participantes la descripción y exploración ${ }^{22,23}$ del proceso de cuidado familiar.

El escenario del estudio estuvo constituido por familias mexicanas del área urbana de la Ciudad de México, que acuden a un dispensario médico ubicado en una colonia popular de la Delegación Gustavo A. Madero; el estudio en esta demarcación ofrece la perspectiva de familias de bajo nivel económico en una área urbana y en su mayoría comerciantes informales.

El trabajo de campo se realizó de noviembre del 2012 a junio del 2013, y después de la autorización se procedió a la revisión de los registros clínicos de familias con adultos mayores, que asistían regularmente al dispensario desde 12 meses antes de iniciado el estudio.

El universo lo constituyeron 57 familias asistentes a este dispensario médico. El diseño muestral que se empleó fue por criterio, lo que permitió reconocer cinco informantes adultos: cuatro mujeres y un hombre entre los 40 y 56 años de edad, no proveedores económicos, responsables de realizar las actividades de cuidado al interior de cada familia y residentes en una colonia periférica al dispensario.

Las familias estaban compuestas por tres y hasta cinco personas, todas con ingreso económico no fijo centrado en un proveedor económico dedicado al comercio informal en mercados ambulantes. Tres de las familias rentan una vivienda, una la tiene prestada y otro es propietario. Las viviendas disponían de dos o tres habitaciones, una empleada como cocina-sala-comedor y la otra como recámara, todos construidos de concreto, paredes y pisos de cemento.

Los datos se recolectaron a través de una guía de entrevista semi-estructurada compuesta por 5 preguntas eje, así como notas de campo; fueron grabadas con autorización de los informantes y transcritas para la validación por cada uno de ellos hasta lograr la aprobación, proceso que fue largo dado que estuvo sujeto a la disponibilidad de tiempo y espacio de los propios informantes. Cada informante y en consecuencia las entrevistas recibieron un pseudónimo a partir de nombres de flores, para asegurar el anonimato.

Inicialmente las entrevistas fueron incidentales y transitaron a profundidad, se llevaron a cabo en el propio domicilio de los informantes, pero, en algunas ocasiones tuvieron que realizarse en espacios como el tianguis donde vendían.

El análisis fue temático e implicó una codificación abierta (análisis descriptivo inicial con el objetivo de denominar temas y subtemas), axial (identificación de las relaciones) y selectiva (integración de los temas en torno a una central) a partir de los discursos recabados, con el propósito de exponer unidades de sentido nuevos y generar categorías empíricas emergentes al respecto del proceso del cuidado familiar ${ }^{23,24}$.

Posteriormente, se procedió a identificar las interrelaciones e interconexiones entre ellas, con el objeto de agruparlas y propiciar la construcción del sentido del dato y proponer la explicación del fenómeno. La construcción de los hallazgos se realizó desde la propuesta teórica de Humberto Maturana, con la Teoría del conocer que explora a los sistemas biológicos, como la familia, en cuanto a su estructura y proceso en las dimensiones autopoiética y de recursividad ${ }^{25}$; mientras que Leonardo Boff desde la Teoría del cuidado esencial ofreció elementos para reconocer los valores que entran en juego ${ }^{26}$. 
Para la interpretación de los datos, el ordenamiento y clasificación mantuvo una profunda inflexión sobre el material en un proceso circular de lo empírico a lo teórico, de lo concreto a lo abstracto y de lo particular a lo general, en busca del significado de pensamientos, sentimientos y comportamientos descritos en los textos, los cuales se contrastaron con las observaciones recuperadas en las notas de campo.

Las consideraciones éticas que acompañaron el proceso investigativo y particularmente la recolección de información, atendieron lo estipulado en la declaración de Helsinki ${ }^{27}$ y el informe Belmont ${ }^{28}$ para preservar la intimidad, la confidencialidad, el anonimato y el respeto del informante de cada familia. Para la aplicación de los principios antes descritos a la conducción de la investigación se consideraron los siguientes requerimientos: consentimiento informado, valoración riesgo-beneficio y selección de los sujetos de investigación conforme la Comisión Nacional para la protección de los sujetos humanos de investigación ${ }^{27,28 .}$

El rigor científico estuvo dado por los principios de auditabilidad, credibilidad y la transferibilidad de Guba y Lincoln ${ }^{29}$ y para asegurar el rigor metodológico, la validez y confiabilidad de la información se atendió a lo propuesto por Morse ${ }^{30}$.

\section{Resultados y discusión}

Las cinco familias de estudio tenían una composición entre dos y cuatro integrantes adultos, todas con un proveedor económico, con ingresos irregulares, comerciantes, con escolaridad primaria incompleta y al menos un adulto mayor con una Enfermedad no transmisible (diabetes y/o hipertensión arterial), atendida desde hace más de un año en el dispensario; cuatro de los informantes eran mujeres y solo un varón; todos residentes en la zona al menos por dos generaciones.

A partir del estudio, se reconoció la categoría Proceso de cuidado familiar, mismo que está sustentado por dos rasgos: la autopoiésis entendido como la capacidad de un sistema de reproducirse y mantenerse por sí mismo ${ }^{25}$, que aplica como proceso autorregulador de lo que ocurre en la unidad viva llamada familia y otro que asegura la movilización del proceso: bucle-recursivo (ver Figura 1) que apunta a mantener, reproducir y adaptar los procesos de cuidado que experimenta la familia, a continuación, se exponen sus implicaciones.

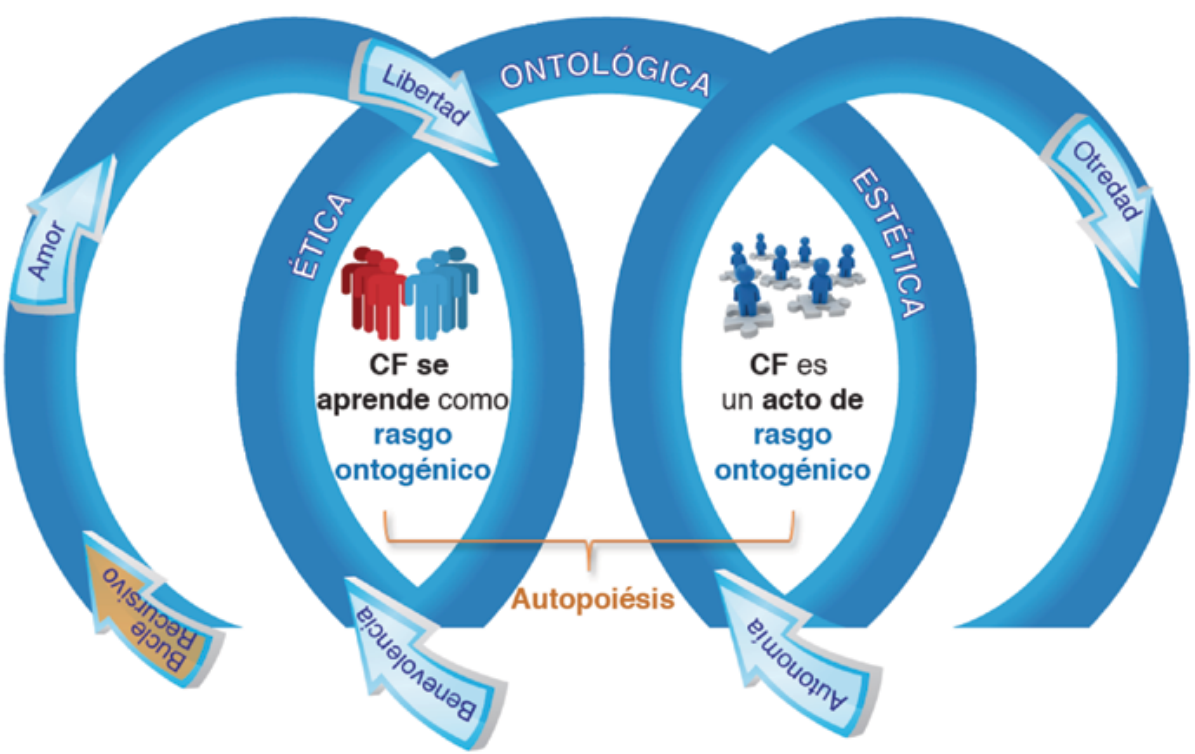

Figura 1. Proceso del Cuidado Familiar

Las familias en forma habitual reproducen y mantienen prácticas de cuidado que con frecuencia los profesionales de la salud consideran barreras para modificar estilos de vida, aunque, parte de la explicación podría ofrecerse desde la consideración de que para las familias el cuidado es un proceso singular y particular que emana de la propia organización como lo refiere Maturana ${ }^{25}$, en tanto las relaciones que se 
establecen entre los integrantes de la misma, incluidas sus prácticas de cuidado, le dan un sentido de pertenencia:

“... por ejemplo, cuando nacen los hijos prácticamente todas las mujeres están reunidas, ya sea porque sean sus hermanas o las esposas de sus hermanos o que sean mis primas o mis cuñadas, ahí están todas [...]. Como que hay ese muégano en la familia y cada una hace lo que ha aprendido desde la casa para cuidar de los nuevos bebés" (Tulipán; 40 años).

La autonomía de los miembros de cada familia abona a la unicidad de la misma, que a través de procesos de interacción construyen y preservan rasgos de cuidado dentro y fuera de la unidad de origen, lo que de algún modo puede explicar la reproducción de ciertas prácticas familiares en materia de salud, bajo la noción de autopoiésis de Maturana.

La preservación del cuidado familiar atiende a un proceso en donde el aprender a cuidar en, para y por la familia se cultiva a través de las generaciones, similar a un rasgo de esencia ontogenénica entendida como conductas aprendidas en la interacción entre los actuantes, para garantizar la permanencia de prácticas de cuidado como resultado de la interacción y comunicación con los otros desde la perspectiva de Maturana ${ }^{31}$. Lo anterior implica ofrecer un esquema de "filtración" para la importación o no de acciones de cuidado desde el exterior, situación que a lo largo de su historia como grupo les permitirá adaptarse a nuevas circunstancias de proveer cuidado a sus miembros.

Desde esta óptica se puede explicar porque las familias cuidan en el fondo con un mismo patrón, pero bajo sus particularidades, aspecto que los prestadores de servicios de salud deben tener presente para acciones de intervención.

"El cuidado de mi familia es y no es igual. Es igual porque en todas las familias nos cuidamos de quienes nos pueden hacer daño, cuidamos a los niños y a los viejos como mi suegra. Y no somos iguales porque cada familia se cuida distinto, como que tenemos puntos de vista diferentes y bueno lo que para una es importante primero para otra es lo último... pero al final es como aprendimos" (Alcatraz, 56 años).

En otras palabras, las familias crean sus configuraciones conductuales con respecto al cuidado, mismas que son ontogénicamente desarrolladas por el grupo, esto como consecuencia de su historia; rasgo que les da un carácter estable y fundamental que se preserva como lo señala Maturana ${ }^{32}$, a continuación, el testimonio que lo refrenda:

“... uno cuida con la vida, no sólo con las manos, pero enseña con el alma para que quede siempre en un lugar donde no se olvide lo bonito de ser cuidado y cuidar al otro con amor" (Margarita, 44años).

La familia es capaz de preservarse, por medio de validar sus valores y sentimientos en la acción cotidiana de cuidar, lo que se traduce en un ejercicio que asegura la continuación de su esencia en el cuidar como familia y que sólo se modificará a partir de la emergencia, es decir un cambio cualitativo del orden familiar, como puede ser la enfermedad, el nacimiento o muerte de algún integrante.

En este marco, el cuidado familiar es un espacio de expresión de las virtudes, que como estructuras ontológicas que el propio grupo genera para afrontar su desarrollo, que le permiten reinterpretar las experiencias de cuidado en el tiempo -definida como auto referente o clausura operativa desde Maturana ${ }^{25,32}$, autoconstruirse constantemente a través del proceso de autopoiésis, en donde como resultado la familia se instaura con un carácter de ser único permanente y universal para cuidar y por tanto, humano.

"Cuidar a una familia no es fácil son muchas cosas, pero en la nuestra es como un eje donde todos cooperamos para que los más chiquitos crezcan y después ellos nos cuiden, porque así debe ser, $[\ldots .$.$] porque les enseñamos a que así debe ser, un ciclo, hoy nos cuidan, mañana cuidamos, para$ que nos vuelvan a cuidar al final de los días; es una oportunidad de aprender, aprenden a ser cuidados, después a cuidar y al final acabamos aprendiendo a dejarnos cuidar" (Bugambilia 43 años).

Por otro lado, al interior la familia y como parte del proceso autopoiético del grupo al cuidar, se reconoce un movimiento de bucle-recursivo, para asegurar la preservación de modelos familiares a través de un acto de 
adaptación, que genera mecanismos de preservación del cuidado como rasgo que identifica al grupo, en donde sus valores determinan la acción cotidiana de cuidar en la familia a través de un proceso de adaptación continuo que se identifica en el discurso siguiente:

“Nos cuidamos como familia porque mamá y papá desde niños nos enseñaron que la familia, los hermanos eran todo, porque lo demás podría moverse, pero la familia no, esa siempre te protegería de lo que fuera. Me acuerdo que los grandes cuidaban a los hermanos pequeños: que no se cayeran, que comieran, que no anduviéramos con las patas chorreadas de pipi... (risas) hasta de no traer los mocos en la cara y a todos nos tocó a mí como hermana mujer los vi a todos, pero el inmediato superior cuidaba a su inmediato inferior, ¡como un ejército... si así...! Pero la que mandaba era mamá y luego yo, hasta la fecha hacemos lo mismo pero diferente, digamos adaptados a los tiempos de hoy" (Bugambilia, 43 años).

El testimonio permite reconocer por un lado que cuidar como proceso al interior de la familia obedece al acto generacional de la misma para reproducir prácticas como parte de un ciclo sin fin (bucle), el sentido de recursividad asegura que algunos rasgos de dichas prácticas familiares para cuidar y ser cuidado, se conserven como consecuencia de su aplicación previa, en otras palabras, el cuidado no es fortuito o espontáneo.

De manera paralela, en ese proceso de preservación generacional, se establece el "sello" del cuidado familiar, que cada grupo social construye y reconstruye para perpetuarse como modo esencial de cuidar como lo apunta Boff ${ }^{26}$.

“... aprendí a cuidar, en el momento no pensé que fuera a proyectar mi vida de una manera tan fuerte, simplemente lo hacía por amor a mi abuela y sí, aprendí que cuidar requería mucho orden, comprometerse y estar si te necesitaba" (Tulipán, 40 años ).

Sin embargo, la familia en su interior y como parte del proceso de cuidar tiene en sí misma un ethos que otorga sentido, dirección e intensidad a las acciones y expresiones del proceso de cuidado al interior del grupo familiar. El ethos ofrece una serie de valores que permiten cuidar y amar como un acto terapéutico y liberador, para con ello dirigir las expresiones del cuidado o resonancias como las llama Boff ${ }^{26}$, como una forma de despejar el futuro y crear esperanzas para sus integrantes ${ }^{33}$, el testimonio señala:

"mis hijas son parte de mi vida pienso que las debo proteger, porque al mismo tiempo son

yo y si ellas son yo y yo soy ellas, entonces nadie mejor para cuidarnos..." (Margarita, 44 años).

En síntesis, al proceso autopoiético del cuidado familiar le subyace un ethos que marca pautas de acción que movilizara en forma de bucle recursivo a la familia, para desarrollar o preservar el cuidado en cada grupo familiar.

\section{Conclusiones}

El reconocimiento de algunos elementos del proceso ontogénico y transgeneracional del cuidado en familias urbanas pobres mexicanas, ofrece a la enfermería contemporánea, por un lado, premisas a considerar desde el punto de vista epistemológico del cuidado en su interior y por otro deja al frente el valor de comprender las relaciones entre los elementos que participan en la preservación, reproducción y potencial modificación de los cuidados en la célula básica ante la posibilidad de intervenir en y con ella, desde la enfermería en particular.

El cuidado familiar es un proceso recursivo determinado por procesos autopoiéticos de sus miembros y de la unidad como tal, en tanto, que sus integrantes experimentan cambios constantes que obedecen a su propio desarrollo e interacción con otros, por lo que se constituye en síntesis social.

También, es una expresión humana resultado de la interacción de la naturaleza humana de sus integrantes, por consiguiente, quien renunciare al cuidado abandonaría su naturaleza humana.

Es evidente que hay valores familiares como el amor lo suficientemente poderoso como para constituirse en un deseo, medio, recurso y expresión que la familia emplea para detonar el movimiento del proceso de cuidar en su interior, aunque resultaría conveniente explorar más el ethos de cuidado en la familia que moviliza o no su desarrollo. 
Finalmente, es necesario seguir explorando el fenómeno del cuidado familiar desde la subjetividad de los actores en diversos escenarios como la cronicidad, la incapacidad, en familias indígenas y urbanas de diferentes niveles económicos, por mencionar algunos en donde la enfermería tiene un espectro de participación importante.

\section{Responsabilidades éticas}

Protección de personas y animales. Los autores declaran que para esta investigación no se han realizado experimentos en seres humanos ni en animales.

Confidencialidad de los datos. Los autores declaran que en este artículo no aparecen datos de pacientes. Derecho a la privacidad y consentimiento informado. Los autores han obtenido el consentimiento informado de los pacientes y/o sujetos referidos en el artículo. Este documento obra en poder del autor de correspondencia.

Financiamiento. Ninguno

Conflicto de intereses. Los autores declaran no tener ningún conflicto de intereses.

\section{Referencias}

1. De la Cuesta C. Cuidado artesanal: la invención ante la adversidad. Medellín: Universidad de Antioquia; 2004: 56.

2. Consejo Nacional de Evaluación de la Política de Desarrollo Social. Medición de la pobreza. Pobreza en México. Resultados de pobreza en México 2016 a nivel nacional y por entidades federativas. México: CONEVAL; 2016. [consultado 1 septiembre 2017]. Disponible en: http://bit.ly/2te20MO

3. Instituto Nacional de Estadística y Geografía. II Conteo de Población y Vivienda 2005. México: INEGI; 2005.

4. Instituto Nacional de Estadística y Geografía. Perspectiva estadística Distrito Federal. Distrito Federal: INEGI; 2012.

5. Observatorio Mexicano de Enfermedades No Transmisibles. Sistema de indicadores para monitorear los avances de la estrategia nacional para la prevención y el control del sobrepeso, la obesidad y la diabetes. México: OMENT; 2017.

6. International Diabetes Federation. Atlas de la diabetes de la FID. Bélgica: Federación Internacional de Diabetes; 2015. P.11-3. [consultado 20 enero 2017]. Disponible en: www.diabetesatlas.org

7. Instituto Nacional de Estadística y Geografía. "Estadísticas a propósito del... día mundial contra el cáncer (4 de febrero)”. Datos nacionales. Aguascalientes: INEGI; 2017. [consultado 6 septiembre 2017]. Disponible en: http://bit.ly/2l8n1QY

8. Oudhof H, Mercado A, Robles E, et al. Características de la familia mexicana contemporánea y su papel en la prevención de la violencia. En: Rojas A, Villalobos G, Brunett K, et al. Por una conducta de paz: una mirada desde las ciencias de la conducta. Estado de México: Universidad Autónoma del Estado de México; 2016.

9. Ramírez E. Enfermería en el pasado y presente de la familia mexicana. Enferm. univ. 2012; 9(9): 57-63.

10. Minuchin S. Familias y Terapia Familiar. Philadelphia: Gedisa; 2009.

11. González C. Teoría estructural familiar. Rancagua: Facultad de CCSS EAPS Psicología, Enfermería y Odontología; 2014.

12. Espinal I, Gimeno A, González F. El enfoque sistémico en los estudios sobre la familia. Revista Internacional de Sistemas. 2006; (14): 21-34.

13. Ibáñez EJ, Vargas JJ, Galván J, et al. Diferenciación en mujeres con papiloma humano: un estudio cualitativo. Revista Electrónica de Psicología Iztacala. 2008; 11 (2): 45-82. [consultado 23 septiembre 2014]. Disponible en: http://bit.ly/2oicGVP 
14. Goldenberg DS. La familia, un paciente olvidado. Rev. Rol enferm. 1997; (223): 25-30.

15. Wright LM, Leahey M. Trends in nursing of families. J Adv Nurs. 1990; 15(2): 148-54.

16. De la Cuesta C. El Cuidado familiar: una revisión crítica. Invest. educ. enferm. 2009; 27 (1): 96102. [consultado 11 julio 2015]. Disponible en: http://bit.ly/2EW4C6S

17. Cuesta C. Familia y cuidados a pacientes crónicos: el papel de la enfermera en el cuidado familiar. Index enferm. 2001; 10(34): 20-6.

18. Pérez B. El cuidado de la enfermería en familia: un reto para el siglo XXI. Aquichan 2002; 2(2): 24-6. [consultado 14 julio 2014]. Disponible en: http://bit.ly/2moGrkh

19. Wallhagen MI, Yamamoto-Mitani N. The meaning of family caregiving in Japan and the United States: a qualitative comparative study. JTranscult Nurs. 2006; 17 (1): 65-73.

20. Wolley DS, Kinner TJ. Comparing perceived self-management practices of adult type 2 diabetic patients after completion of a structured ADA certified diabetes self-management education program with unstructured individualized nurse practitioner led diabetes self-management education. Appl Nurs Res. 2016; (32): 171-6. https://dx.doi.org/10.1016/j.apnr.2016.07.012

21. Jutterström L; Hörnsten A, Sandström H, et al. Nurse-led patient-centered self-management support improves HbA1c in patients with type 2 diabetes-A randomized study. Patient Educ Couns. 2016; 99(11): 1821-9. https://dx.doi.org/10.1016/j.pec.2016.06.016

22. De Souza-Minayo MC. La artesanía de la Investigación cualitativa. Buenos Aires: Lugar Editorial; 2009.

23. Hernández R, Fernández C, Baptista P. Metodología de la Investigación. $5^{a}$ ed. CDMX: McGraw-Hill Interamericana; 2010.

24. De Souza-Minayo MC. Investigación Social. Teoría, Método y Creatividad. Buenos Aires: Lugar Editorial; 2003.

25. Maturana H, Varela F. El árbol del conocimiento. Las bases biológicas del conocimiento humano. Buenos Aires: Lumen/Editorial Universitaria; 2003.

26. Boff L. El Cuidado Esencial. Ética de lo Humano. Compasión por la tierra. Madrid: Trotta; 2002.

27. Asociación Médica Mundial. Declaración de Helsinki de la AMM-Principios éticos para las investigaciones médicas en seres humanos. Ginebra: AMM; 2017.

28. Comisión Nacional para la protección de los sujetos humanos de investigación biomédica y comportamental. Informe Belmont. Principios y guías éticos para la protección de los sujetos humanos de investigación. Pamplona: National Institutes of Health; 2003.

29. Guba EG, Lincoln YS. Effective evaluation. Improving the usefulness of evaluation results through responsive and naturalistic approaches. San Francisco: Jossey-Bass; 1981.

30. Morse JM, Barrett M, Mayan M, et al. Verification strategies for establishing reliability and validity in qualitative research. Int J Qual Methods. 2002; 1(2): 13-22.

31. Maturana H. La realidad: ¿Objetiva o construida? I. Fundamentos biológicos de la realidad. Barcelona: Anthropos/Universidad Iberoamericana/ITESO; 1995.

32. Maturana H, Pörksen B. Del Ser al Hacer. Los orígenes de la biología del conocer. Santiago de Chile: Lom Ediciones; 2004.

33. Boff L. El ethos que cuida. Servicios Koinonia, 2003. [consultado 7 julio 2012]. Disponible en: http://bit.ly/2oTxz9C 